Converse mode piezoelectric coefficient for lead zirconate titanate thin film with interdigitated electrode

This content has been downloaded from IOPscience. Please scroll down to see the full text. 2015 J. Micromech. Microeng. 25045016 (http://iopscience.iop.org/0960-1317/25/4/045016)

View the table of contents for this issue, or go to the journal homepage for more

Download details:

IP Address: 128.179.132.76

This content was downloaded on 24/03/2015 at 10:38

Please note that terms and conditions apply. 


\title{
Converse mode piezoelectric coefficient for lead zirconate titanate thin film with interdigitated electrode
}

\author{
N Chidambaram, D Balma, R Nigon, A Mazzalai, R Matloub, C S Sandu \\ and $\mathbf{P}$ Muralt \\ Laboratoire de Céramique, École Polytechnique Fédérale de Lausanne EPFL, Lausanne, Switzerland \\ E-mail: c.nachiappan@gmail.com
}

Received 21 October 2014, revised 12 January 2015

Accepted for publication 27 January 2015

Published 23 March 2015

\begin{abstract}
The use of interdigitated electrodes (IDEs) in conjunction with ferroelectric thin films shows many attractive features for piezoelectric MEMS applications. In this work, growth of $\left\{\begin{array}{lll}1 & 0 & 0\end{array}\right\}$-textured lead zirconate titanate (PZT) thin films was achieved on insulating $\mathrm{MgO}$ buffered, oxidized silicon substrates. IDEs were fabricated by lift-off techniques and cantilevers were formed by dicing. The deflection upon application of a sweeping voltage was measured as large signal response in parallel to the ferroelectric polarization (PV loop). Likewise, the small signal piezoelectric response was measured in parallel to the capacitancevoltage (CV) measurement. In this way, a complete picture of the ferroelectric-piezoelectric element was obtained. From the deflection, the in-plane piezoelectric stress in the PZT thin film was derived and, from this, the effective piezoelectric coefficients. For the latter, two types were defined: an engineering type corresponding to the average value along the IDE, which can directly be compared to coefficient of a parallel plate electrode (PPE) capacitor and a second one that approximately yields the idealized coefficient governing between the electrode fingers. The IDE structures were experimentally compared with PPE structures of identical film thickness. The resulting coefficients were of opposite sign, as expected. In spite of a much better polarization loop, the IDE device showed a lower average piezoelectric stress. The estimated peak value between the fingers was about the same as in the PPE device, corresponding to about $20 \mathrm{C} \mathrm{m}^{-2}$. Nevertheless, the result is very promising for cases where compressive piezoelectric stresses are required and for preventing cracking due to large piezoelectric tensile stresses in PPE systems.
\end{abstract}

Keywords: ferroelectric, piezoelectric, PZT thin films, $e_{31, f}, e_{33}$, if

(Some figures may appear in colour only in the online journal)

\section{Introduction}

The interest in piezoelectric MEMS is rapidly increasing due to a variety of potential applications as micro-actuators [1-4], energy harvesting devices [5,6] and, lately, in logic gates as piezo-electronic transistors (PETs) for low power consumption [7]. Most of these applications utilize micro-machined membranes, bridges or cantilever structures, which are laminated with a piezoelectric film having its electrodes in a parallel plate electrode (PPE) configuration. Such structures utilize the transverse piezoelectric effect exerting an in-plane stress upon application of an electric field, governed by an effective piezoelectric coefficient $e_{31, f}$ being different from $e_{31}$ due to the non-zero strain perpendicular to the film plane ( $e_{31}$ is defined for a completely clamped situation), first derived by Muralt et al $[8,9]$. There is another possible electrode configuration: interdigitated electrodes (IDEs). This electrode system is well known for exciting surface acoustic waves or Lamb waves [10]. This configuration can also be used for MEMS actuators (see, e.g. [11]) when we deal with ferroelectric thin films such 


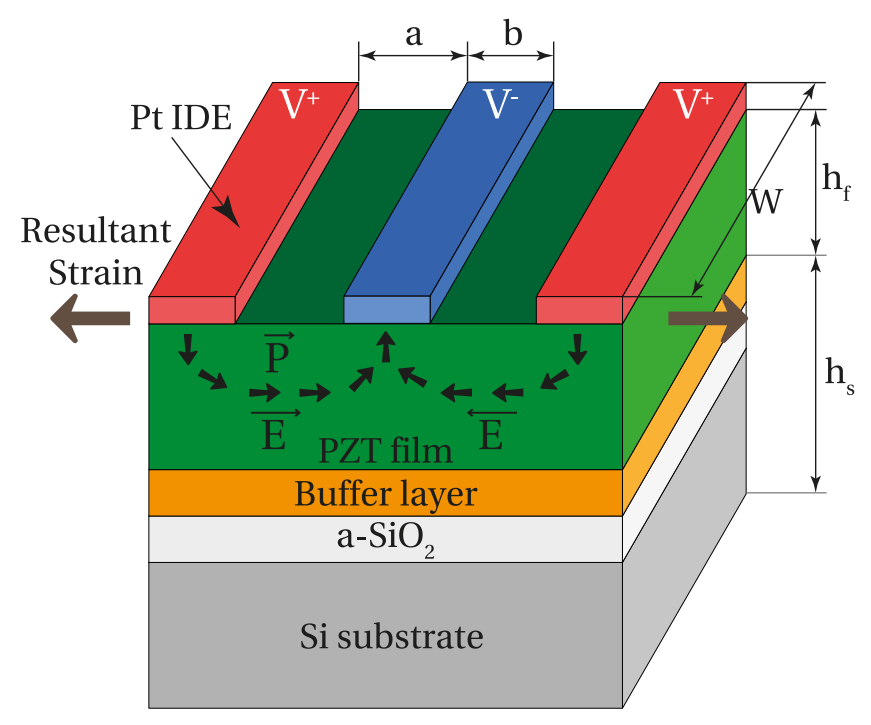

Figure 1. Schematic drawing of PZT thin film stack (showing geometric parameters) in an interdigitated electrode system. Positive $(V+)$ and negative $(V-)$ voltages are applied to the electrode fingers. The ferroelectric polarization follows the electric field above the coercive field, or due to poling, as indicated by the small arrows inside PZT. The resulting piezoelectric in-plane strain is indicated by the thick arrows. If the strain is assumed to be zero, resultant blocking-stress from substrate is compressive.

as lead zirconate titanate (PZT) thin films. Through a poling process, the polarization between the electrode fingers is alternating its direction in-phase with the electric field and thus the product $\vec{P} \cdot \vec{E}$ is everywhere positive (ideally), leading to a compressive stress, being the term $-e_{33} E_{3}$ dominant between the fingers (see figure 1). As we will show, it is indeed an effective longitudinal piezoelectric coefficient (which is different from $e_{33}$ ) that describes the created in-plane stress for a given electric field. This is interesting because of three reasons: (1) the stress has opposite sign as compared to the PPE configuration, i.e. compressive instead of tensile. This results in the opposite curvature of cantilevers. These features might be interesting for certain applications. (2) Ceramic materials are prone to cracking at high tensile stress levels. Hence, with IDEs, higher values of in-plane piezoelectric stresses are conceivable, as they are compressive. (3) The longitudinal effect $\left(e_{33}\right)$ is about three times larger than the transverse effect $\left(e_{31}\right)$, considering data of PZT 4 ceramics. One of the complications using IDEs is the requirement to grow the ferroelectric thin films on an insulating substrate. One cannot profit from the vast experience to grow $\{100\}$ textured lead based perovskite thin films on platinum (111) electrodes. Instead, growth must start from amorphous thermal oxide, the most convenient insulating film to isolate from potentially conducting silicon substrates, or device layers.

In this work, we studied the growth of textured PZT thin films on a suitable dielectric buffer layer on the thermal oxide of a silicon substrate and fabricated IDE structures on PZT thin films. In earlier articles on the direct effect useful for energy harvesting $[12,13]$, we identified the $\{100\}$ texture ideal for tetragonal films and the $\{110\}$ texture ideal for rhombohedral thin films. The $\{111\}$ texture is not desired in both cases. In case of morphotropic phase boundary composition, a mixture of

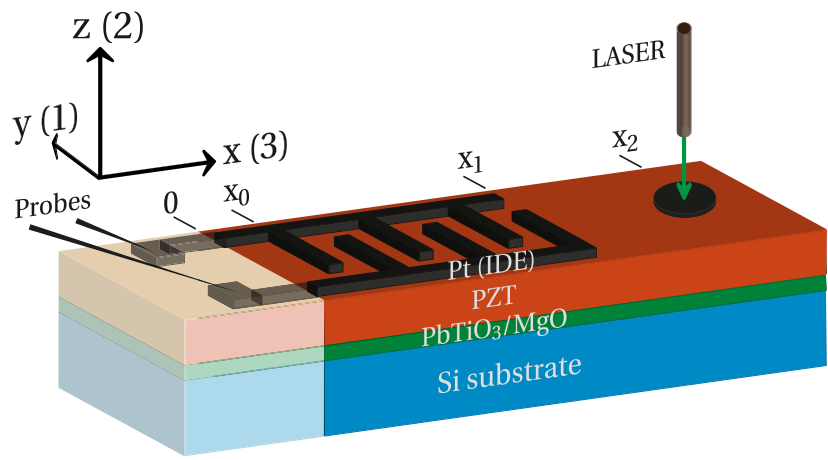

Figure 2. Schematic illustration of PZT with IDE system, showing the coordinates $x, y, z$ representing the cantilever frame and coordinates $1,2,3$ representing the piezoelectric material with 3 indicating the poling direction. Translucent part represents the clamped portion of the cantilever.

$\{100\}$ and $\{110\}$ might be optimal. As dielectric buffer layer, most of the research groups have used zirconia $\left(\mathrm{ZrO}_{2}\right)$ barrier layers to grow PZT on oxidized silicon. This is very efficient in preventing lead diffusion, but does not promote texturing of PZT [11, 14-19]. We found that evaporated magnesia (MgO) thin films worked well as dielectric buffer layers promoting $\{100\}$ growth. In order to keep the electric field as much as possible parallel to the substrate plane, we used relatively thin PZT films of only $500 \mathrm{~nm}$. We elaborated methods to assess the piezoelectric effect of IDE systems in the converse mode (i.e. actuator mode), similar to the ones for PPE structures [20, 21]. The converse effect can be measured as large signal response, as opposed to the usual methods for the direct effect that measure a small signal response [22-24]. Large signal measurements are more relevant for actuators requiring large strokes. For comparison and illustration we compared the performance of the IDE system with the PPE system having identical PZT film thickness.

\section{Fabrication and characterization methods}

$500 \mathrm{~nm}$ sol-gel gradient free MPB PZT based on the chemical route devised by Gurkovich and Blum [25] were deposited on top of e-beam evaporated $\mathrm{MgO}$ buffer layer (at $200{ }^{\circ} \mathrm{C}$ ) with a thin layer of $\mathrm{PbTiO}_{3}$ seed layer. This process was optimized for lead excess and composition in each layer to get gradient free PZT [26, 27]. The substrate being (100) silicon (Si) wafer with $4 \mu \mathrm{m}$ thick wet oxide of silicon. Platinum (Pt) was sputtered on top of PZT and IDE structures were obtained by standard lithographic lift-off process. Similar deposition was carried out with PPE configuration with $\mathrm{Pt}(100 \mathrm{~nm}) / \mathrm{TiO}_{2}(30 \mathrm{~nm}) /$ Ti $(3 \mathrm{~nm})$ bottom electrode on oxidized silicon $(500 \mathrm{~nm}$ wet oxide). Sputtered platinum top electrode was obtained by liftoff here as well. Before any electrical measurements, the wafer was annealed at $550{ }^{\circ} \mathrm{C}$ in oxygen atmosphere to remove the sputter damage at the Pt/PZT interface. X-ray diffraction data were collected with a Bruker D8 Discover diffractometer using monochromatic $\mathrm{CuK}_{\alpha}$ radiation. TEM cross section was analysed with FEI Tecnai Osiris microscope with $200 \mathrm{kV}$ accelerating voltage. The piezoelectric coefficients were measured by applying a voltage and measuring the displacement at the tip of cantilever $(15 \mathrm{~mm} \times 1.5 \mathrm{~mm} \times 0.5 \mathrm{~mm})$ by single beam laser 


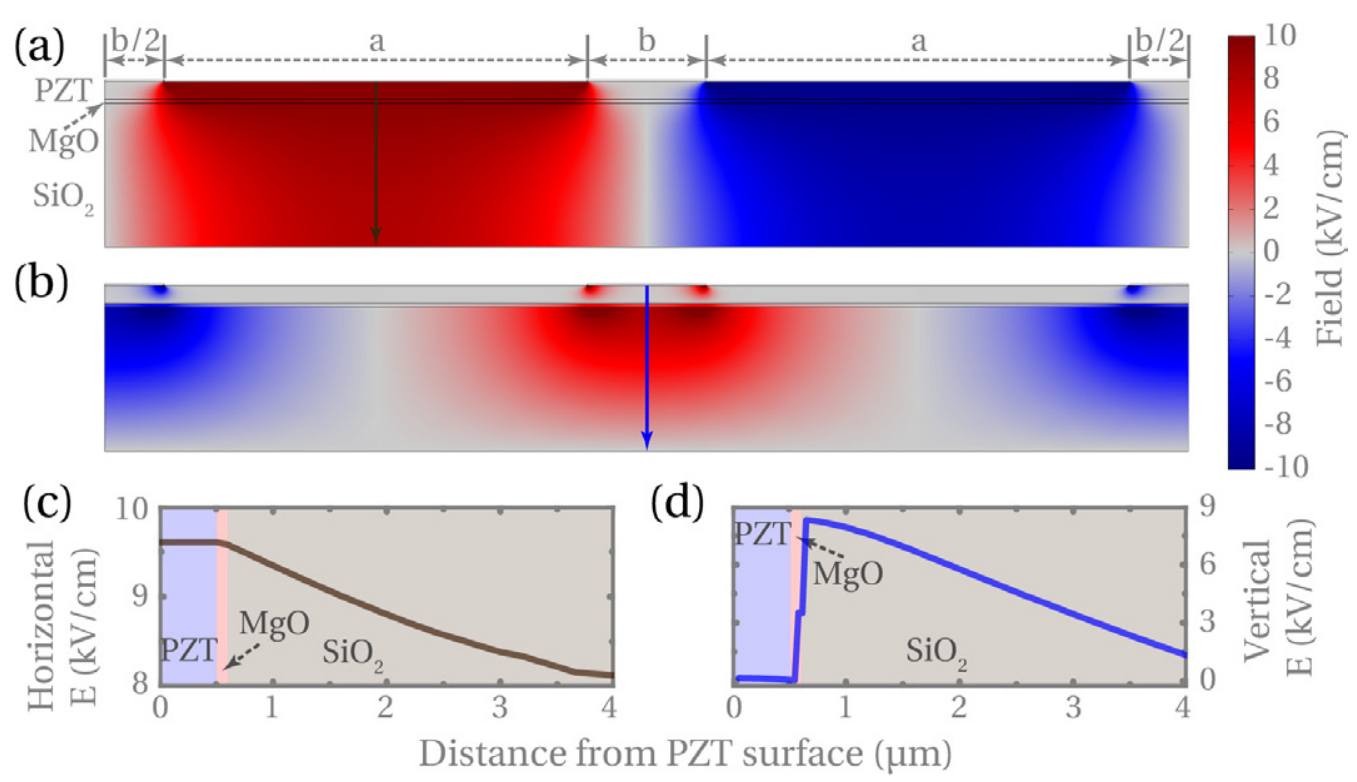

Figure 3. Finite element simulation results showing the field distribution in the sample when a nominal V/a of $10 \mathrm{kV} \mathrm{cm}{ }^{-1}$ is applied. The lateral dimensions are: $a=13 \mu \mathrm{m}, b=3.5 \mu \mathrm{m}$. (a) The value of the horizontal component of the field; $(b)$ the value of the vertical component of the field; $(c)$ the horizontal component in the mid plane between the IDE fingers (brown arrow in $(a)$ ) as a function of depth; and $(d)$ the vertical component at the mid plane below the electrode (blue arrow in $(b)$ ).

interferometer (figure 2), the long axis of the cantilevers is parallel to the [1 10$]$ direction of the silicon crystal. An aixACCT TF Analyzer 2000 tool, coupled with a SIOS SP-S laser interferometer, was used for measuring the tip displacement along with polarization loop (PV) and capacitance loop (CV) measurement. The piezoelectric coefficient is derived from the bending of a cantilever structure upon application of an electric field. A schematic of the employed cantilevers is shown in figure 2. We have chosen two coordinate systems, one for the PZT thin film for complying with the polarization in the plane along the 3-direction and one for the cantilever having its long axis along $x$ and the vertical growth direction along $z$.

\section{FEM simulation of the electric field}

In order to get a clear idea about the electric field distribution, 2D finite element simulations were performed for the actual geometry, i.e. $500 \mathrm{~nm}$ of PZT on $100 \mathrm{~nm} \mathrm{MgO}$ buffer layer on $4 \mu \mathrm{m}$ of silicon dioxide layer. Below the latter, no material was assumed to be present (vacuum). A COMSOL Multiphysics software package was utilized. The field was calculated for a voltage of $V=13 \mathrm{~V}$, corresponding to an approximate electric field of $V / a=10 \mathrm{kV} \mathrm{cm}^{-1}$. We assumed an infinite number of fingers to model the field lines, meaning that we designed a pair of fingers in 2D (unit structure of IDE) and applied periodic boundary conditions to the left and right boundaries. To simplify the modeling, the PZT layer was approximated by a layer of an isotropic dielectric material of dielectric constant 1000 , close to the actual value measured for the IDE case at zero field. A dielectric constant of 10 was used for $\mathrm{MgO}$ and the built-in dielectric constant of 4.2 was used for the $\mathrm{SiO}_{2}$ layer. The assumption that PZT behaves like an isotropic dielectric material is the same approximation as made for conformal mapping method used by Gevorgian et al [28].
The software solves the equation of electrostatics at the nodes and extrapolates the variables within each element. The meshing was generated by COMSOL, only its density was set manually. Figures $3(a)$ and $(b)$ show the horizontal and vertical component of the electric field, respectively. The horizontal component is concentrated between the electrode fingers and the vertical component exists only near the edge of the electrode fingers. It is interesting to note that there is almost no electric field below the IDE fingers (both horizontal and vertical component). Figure 3(c) shows the horizontal electric field component from the top surface of PZT till the bottom of $\mathrm{SiO}_{2}$ at the mid plane between the fingers. One can see that the field is quite homogeneous through the PZT layer, but $4 \%$ smaller than the nominal field V/a. Below PZT, the field decreases gradually as we move through $\mathrm{SiO}_{2}$. Figure $3(d)$ shows the vertical electric field component along a vertical line going through the center of the electrode. There is only a very small electric field in the PZT region, followed by an upward jump at the $\mathrm{MgO}$ buffer layer and then a gradually decreasing field in the $\mathrm{SiO}_{2}$ region. The large jump is due to the requirement of a continuous electrical displacement field $\mathrm{D}$ and thus to the large difference in the dielectric constants. Since in addition, the thickness of the $\mathrm{SiO}_{2}$ layer is larger than the one of the PZT layer, the very main drop of voltage occurs in the $\mathrm{SiO}_{2}$ layer and thus the vertical electric field in PZT is practically zero.

\section{Material piezoelectric coefficient in an idealized structure}

We consider constitutive equations in between two fingers (idealized case: IDE finger width $b \rightarrow 0$, see figure 1), where the electric field and thus the average polarization (after poling) are in the plane along the 3 -axis. The macroscopic 
symmetry is orthorhombic as the 1 and 2 directions are not equivalent (as the out of plane direction is un-clamped). Microscopically, the two directions are also different as one is the growth direction and we have columnar grains with gradient in composition along the growth direction. We derive the idealized piezoelectric $\hat{e}$ coefficient (symbolized with an over-hat) from the following constitutive equations, that are valid for poling along the direction 3 :

$$
\begin{gathered}
\widehat{T}_{1}=c_{11}^{E} \xi_{1}+c_{12}^{E} \xi_{2}+c_{13}^{E} \xi_{3}-\widehat{e_{31}} E_{3} \\
\widehat{T}_{2}=c_{12}^{E} \xi_{1}+c_{22}^{E} \xi_{2}+c_{23}^{E} \xi_{3}-\widehat{e_{32}} E_{3} \\
\widehat{T}_{3}=c_{13}^{E} \xi_{1}+c_{23}^{E} \xi_{2}+c_{33}^{E} \xi_{3}-\widehat{e_{33}} E_{3} \\
\widehat{D_{3}}=\widehat{e_{31}} \xi_{1}+\widehat{e_{32}} \xi_{2}+\widehat{e_{33}} \xi_{3}+\epsilon_{33}^{S} E_{3}
\end{gathered}
$$

Thin film is free to move in the out-of-plane direction, meaning that $T_{2}$ is 0 . By definition we consider perfect clamping (infinitely rigid substrate), so that the in-plane strains ( $\xi_{1}$ and $\xi_{3}$ ) and can be equated to zero. It follows that the piezoelectric in-plane stress along the $x=3$ axis is computed to be the following:

$$
\begin{gathered}
\widehat{T_{3}}=-\left[\widehat{e_{33}}-\frac{c_{23}^{E}}{c_{22}^{E}} \widehat{e_{32}}\right] E_{3} \\
\widehat{T_{3}}=-\widehat{e_{33, i f}} E_{3}
\end{gathered}
$$

The term inside the square bracket in equation (5), represents the idealized piezoelectric coefficient measured in converse mode with the IDE. We call this effective piezoelectric $e$ coefficient for IDEs, $\widehat{e_{33, i f}}$ (equation (6)). In order to derive the resulting constitutive equation in the direct mode following calculations are derived (from equation (2)):

$$
\xi_{2}=\frac{1}{c_{22}^{E}}\left[\widehat{e_{32}} E_{3}-c_{12}^{E} \xi_{1}-c_{23}^{E} \xi_{3}\right]
$$

and applying equations (7) in (4), we get:

$$
\begin{gathered}
\widehat{D_{3}}=\left[\widehat{e_{31}}-\frac{c_{12}^{E}}{c_{22}^{E}} \widehat{e_{32}}\right] \xi_{1}+\left[\widehat{e_{33}}-\frac{c_{23}^{E}}{c_{22}^{E}} \widehat{e_{32}}\right] \xi_{3}+\left[\epsilon_{33}^{S}+\frac{{\widehat{e_{32}}}^{2}}{c_{22}^{E}}\right] E_{3} \\
\widehat{D_{3}}=\left[\widehat{e_{31, i f}}\right] \xi_{1}+\left[\widehat{e_{33, i f}}\right] \xi_{3}+\left[\widehat{\epsilon_{33, i f}} \epsilon_{0}\right] E_{3}
\end{gathered}
$$

In principle, directions 1 and 2 are not equivalent but we can nevertheless state that directions 1 and 2 must be approximately equivalent so the effective orthorhombic symmetry should be close to a tetragonal one. Using standard PZT-4 parameters, $e_{31, i f}$ is calculated as $-1.9 \mathrm{C} \mathrm{m}^{-2}$, a smaller absolute value than the $-4.1 \mathrm{C} \mathrm{m}^{-2}$ of $e_{31}$ for PZT-4. However, $e_{33 \text {, if }}$ is larger than $e_{33}: 16.3$ versus $14.1 \mathrm{C} \mathrm{m}^{-2}$. Similar conclusions on this large anisotropy were made in [29] (equation (5)). The signs of these corrections arise due to fact that the stiffness coefficients are all positive and $e_{31}$ is negative. Such a large difference in the in-plane piezoelectric coefficients is in fact very suitable for bending cantilevers: the bending along the desired, long direction is much stronger than the transverse bending. For comparison, the same PZT-4 materials parameters yield a transverse piezoelectric coefficient for the PPE geometry of $e_{31, f}=-13 \mathrm{C} \mathrm{m}^{-2}$. This means that IDEs would show a larger coefficient between the fingers, but that this advantage would be reduced by the width of the fingers. We roughly estimate that the effective (engineering) coefficient would turn out to be: $e_{33, i f} \cdot \frac{a}{a+b}=12.8 \mathrm{C} \mathrm{m}^{-2}$ (with $a=3.5$ and $b=13 \mu \mathrm{m})$. We thus would expect the same excursion of our cantilevers at identical electric fields, but of opposite sign.

The direct effect can be deduced from equation (8), as shown in an earlier paper [12]. Considering that the strains are coupled through the Poisson ratio $\xi_{1}=-\nu_{s} \xi_{3}$ and in absence of an electric field $\left(E_{3}=0\right)$ and assuming equivalence of 1 and 2 directions $\left(e_{31}=e_{32}\right)$, the earlier direct mode coefficient is again obtained as follows:

$$
\begin{gathered}
\widehat{D_{3}}=\left\{\widehat{e_{31}}\left[\nu_{s} \frac{c_{12}^{E}}{c_{11}^{E}}-\frac{c_{13}^{E}}{c_{11}^{E}}-\nu_{s}\right]+\widehat{e_{33}}\right\} \xi_{3} \\
\widehat{D_{3}}=\widehat{e_{\mathrm{IDE}}} \xi_{3}
\end{gathered}
$$

\section{Beam bending in an IDE system}

In order to calculate the deflection of IDE cantilevers, we consider the following cases: (1) the silicon substrate is thicker than a period of the IDE, i.e. $a+b$ (see figure 1), resulting in a rather constant curvature, which will increase with the ratio $a / b$. This is the case we are actually dealing with in our measurements with cantilevers having the full wafer thickness. Additionally, we use the 'nominal' electric field of V/a to derive the coefficient from the in-plane piezoelectric stress. So obtained piezoelectric coefficient is not a true material property, but describes the effective piezoelectric behavior of such a beam. We shall call it the engineering coefficient. It can be directly compared to the coefficient governing the PPE configuration. (2) The idealized piezoelectric coefficient, presented in equation (6), approximating the real coefficient between the fingers, can be calculated from the measured engineering coefficient by multiplication with the product $(a+b) / a$ and corrected for the somewhat lower field actually present between the fingers as compared to the nominal field (in above simulation, 96\%). Following the results of the finite element calculation, we include the fact that the electric field below the fingers is zero also in the vertical component. This means that there is no transverse effect that would produce an opposite piezoelectric stress. In this second case, we still assume that the substrate is thicker than the IDE period. (3) The substrate thickness is comparable with the dimensions of $a, b$ and $h_{f}$, different curvatures of the cantilever are expected in between the IDE fingers and below the IDE fingers. In this case, the bending radius is not anymore constant over the period of the IDE. It is governed by the same piezoelectric coefficient as case (2) between the fingers and the zero electric field giving zero bending below the electrodes. The averaging of the bending is not precisely identical to case (1), meaning that the derivation of the coefficient from the cantilever bending is 
Table 1. Description of various piezoelectric coefficients and piezoelectric stress for the IDE system.

\begin{tabular}{|c|c|c|}
\hline Coefficient abrev. & Description & Case \\
\hline$\widehat{e_{33, i f}}, \widehat{T_{3}}$ & Theoretical value for idealized case. & $\begin{array}{l}\text { Out-of-plane un-clamped } \\
\text { coefficient for homogeneous } \\
E_{3} \text { field strictly along } x \text { axis }\end{array}$ \\
\hline$\overline{e_{33, i f}}, \overline{T_{3}}$ & $\begin{array}{l}\text { Describes the average } \\
\text { piezoelectric in-plane stress, bending radius constant. } \\
\text { Allows direct comparison with PPE case. }\end{array}$ & $h_{s}>a+b$ \\
\hline$\widetilde{e_{33, i f}}, \widetilde{T_{3}}$ & $\begin{array}{l}\text { Describes bending radius between the fingers. } \\
\text { Approximately equal to } \widehat{e_{33, i f}} \text { after correcting for electric field. }\end{array}$ & $h_{s}<a+b$ \\
\hline
\end{tabular}

somewhat different. Although we don't have this case with our cantilever thickness, we have presented the formulation which could be useful for micromachined substrates. A summary of various piezoelectric coefficients for the IDE system is listed in table 1. Derivation for each of these coefficients will be presented in the following subsections.

\subsection{Engineering approximation}

Let us consider first an effective value of the piezoelectric coefficient, effective in the sense that it causes an average curvature along the coverage of the IDE (length $=2 N(a+b)$ ), where $\mathrm{N}$ is the number of pairs of electrodes. Uniform curvature scenario is true in the case when $\left(h_{f}, a, b\right) \ll h_{s}$. These coefficients can be directly compared to the $e_{31, f}$ value of a PPE system. This average curvature is negative. The curvature is linearly amplified in the region between $x_{1}$ (end of the IDE) and $x_{2}$, where the displacement is measured (see figure 2). It should be noted that we have made $x_{0}$ to be 0 by experimental design. So in the IDE coverage, the average curvature $(1 / \bar{R})$ can be written as the second derivative of displacement function $u(x)$ :

$$
\frac{1}{\bar{R}}=\frac{\mathrm{d}^{2} u(x)}{\mathrm{d} x^{2}}
$$

Displacement at $x_{1}$ can be calculated by integrating equation (12):

$$
u\left(x_{1}\right)=\frac{x_{1}^{2}}{2 \bar{R}}
$$

Displacement at $x_{2}$ can be found by adding the product of slope at $x_{1}$ (which is $\left.x_{1} / \bar{R}\right)$ and the distance $\left(x_{2}-x_{1}\right)$ to equation (13):

$$
u\left(x_{2}\right)=\frac{x_{1}\left(2 x_{2}-x_{1}\right)}{2 \bar{R}}
$$

From equation (14), the average radius of curvature $(\bar{R})$ can be calculated from a known displacement at $x_{2}$. This curvature can be implemented in Stoney's equation (shown in equation (15) [30]) to get in-plane stress.

$$
T_{x}=\frac{1}{6} \frac{Y_{s}^{\prime} h_{s}^{2}}{h_{f}} \frac{1}{R}
$$

where $Y^{\prime}{ }_{s}$ is the effective modulus of the substrate. In the IDE case, the in-plane directions are different and one expects more deformation along the longer axis of the cantilever where the electric field is applied, so it will be more suitable to simply use Young's modulus for the IDE case. Therefore we get: average blocking in-plane stress $\left(\bar{T}_{3}\right)$

$$
\overline{T_{3}}=\frac{Y_{s} h_{s}^{2}}{3 h_{f} c_{f}} \cdot \frac{u\left[x_{2}\right]}{x_{1}\left(2 x_{2}-x_{1}\right)}=-\overline{e_{33, i f}} \cdot E_{3}
$$

Here $c_{f}$ is the electrode width coverage factor which is simply the ratio of IDE width $W$ to the total cantilever width. And slope of this average in-plane stress generated with respect to applied electric field is average $-\overline{e_{33, i f}}$ (from equation (6)).

\subsection{Deriving an approximation for the material piezoelectric coefficient}

From case 1, we can derive an approximate value for the idealized $\widehat{e_{33, i f}}$ in between the electrode fingers presented in equation (6). FEM results indicate that there is no vertical component of electric field below the IDE finger, so we can safely neglect any piezoelectric stress that can be caused by transverse piezoelectric coefficient. In the following equation V/a is the approximate applied electric field and $E_{3}$ is the nominal horizontal component of electric field in the PZT film between the electrodes. The fraction of approximate electric field and nominal field (in equation (18)) is identified as 1.04 from FEM simulations.

$$
\begin{gathered}
\overline{e_{33, i f}} \cdot \frac{V}{a}=\frac{a}{a+b} \widehat{e_{33, i f}} \cdot E_{3} \\
\widehat{e_{33, i f}}=\overline{e_{33, i f}} \cdot \frac{a+b}{a} \cdot \frac{V / a}{E_{3}}
\end{gathered}
$$

\subsection{Materials coefficient for the thin substrate case}

In this case, the curvature of the beam is modulated and adapts with the PZT film. We make the following assumptions: (1) A constant curvature (radius $R$ ) proportional to the applied electric field is created between the fingers. (2) In the regions below the fingers, no curvature is imposed (dead zone). At the boundaries of the electrodes, the deflection function $u(x)$ is continuous, as well as its derivative $\mathrm{d} u / \mathrm{d} x$. For the IDE system, the $x$ axis can be represented as, $x=n(a+b)+x_{n}$. Where $n$ is a number increasing from 0 to $N^{\prime}\left(N^{\prime}\right.$ is twice the number of pairs of IDEs). $a$ is the gap between electrode fingers, $b$ the 
width of a single IDE finger and $x_{n}$ ranging from 0 to $a+b$. Based on the previous statements, we can write the following:

$$
\begin{gathered}
\frac{\mathrm{d}^{2} u(x)}{\mathrm{d} x^{2}}=\frac{1}{\widetilde{R}} \quad \text { if } \quad 0<x_{n}<a \\
\frac{\mathrm{d}^{2} u(x)}{\mathrm{d} x^{2}}=0 \quad \text { if } \quad a<x_{n}<(a+b)
\end{gathered}
$$

From this curvature information, one can derive the slope and displacement as:

$$
\frac{\mathrm{d} u(x)}{\mathrm{d} x}[x=n(a+b)]=u_{n}^{\prime}=u_{n-1}^{\prime}+\frac{a}{\widetilde{R}}
$$

$$
\begin{aligned}
& u(x)[x=n(a+b)]=u_{n}=u_{n-1}+u_{n-1}^{\prime} \cdot(a+b)+\frac{a^{2}}{2 \widetilde{R}} \\
& +\frac{a b}{\widetilde{R}}
\end{aligned}
$$

The sum of all slopes below IDE can be written as:

$$
u_{n}^{\prime}=\frac{n \cdot a}{\widetilde{R}}
$$

From equations (22) and (23), we can write:

$$
\begin{gathered}
u_{n}=u_{n-1}+\frac{(n-1) a}{\widetilde{R}}(a+b)+\frac{a^{2}}{2 \widetilde{R}}+\frac{a b}{\widetilde{R}} \\
u_{n}=\sum_{n=1}^{N^{\prime}} \frac{(n-1) a}{\widetilde{R}}(a+b)+\frac{a^{2}}{2 \widetilde{R}}+\frac{a b}{\widetilde{R}} \\
=\frac{N^{\prime}\left(N^{\prime}-1\right) a}{2 \widetilde{R}}(a+b)+\frac{N^{\prime} a^{2}}{2 \widetilde{R}}+\frac{N^{\prime} a b}{\widetilde{R}}
\end{gathered}
$$

Therefore the displacement at $x_{1}$ (figure 2) can be simplified from (26):

$$
u\left[x_{1}\right]=\frac{N^{\prime 2} a^{2}}{2 \widetilde{R}}\left[1+\left(1+\frac{1}{N^{\prime}}\right) \frac{b}{a}\right]
$$

The displacement at $x_{2}$ can be written as:

$$
\begin{gathered}
u\left[x_{2}\right]=u\left[x_{1}\right]+\left(x_{2}-x_{1}\right) \frac{\mathrm{d} u(x)}{\mathrm{d} x}\left[x_{1}\right] \\
=\frac{N^{\prime 2} a^{2}}{2 \widetilde{R}}\left[1+\left(1+\frac{1}{N^{\prime}}\right) \frac{b}{a}\right]+\left(x_{2}-x_{1}\right) \frac{N^{\prime} a}{\widetilde{R}}
\end{gathered}
$$

From equation (29), the radius of curvature is calculated to be the following:

$$
\frac{1}{\widetilde{R}}=\frac{u\left[x_{2}\right]}{\frac{N^{\prime 2} a^{2}}{2}\left[1+\left(1+\frac{1}{N^{\prime}}\right) \frac{b}{a}\right]+\left(x_{2}-x_{1}\right) N^{\prime} a}
$$

Equation (30), can be applied in Stoney's formula shown in equation (15) to get the in-plane stress developed:

$$
\widetilde{T}_{3}=\frac{Y_{s} h_{s}^{2}}{6 h_{f} c_{f}} \cdot \frac{u\left[x_{2}\right]}{\frac{N^{\prime 2} a^{2}}{2}\left[1+\left(1+\frac{1}{N^{\prime}}\right) \frac{b}{a}\right]+\left(x_{2}-x_{1}\right) N^{\prime} a}=-\widetilde{e_{33, i f}} \cdot E_{3}
$$

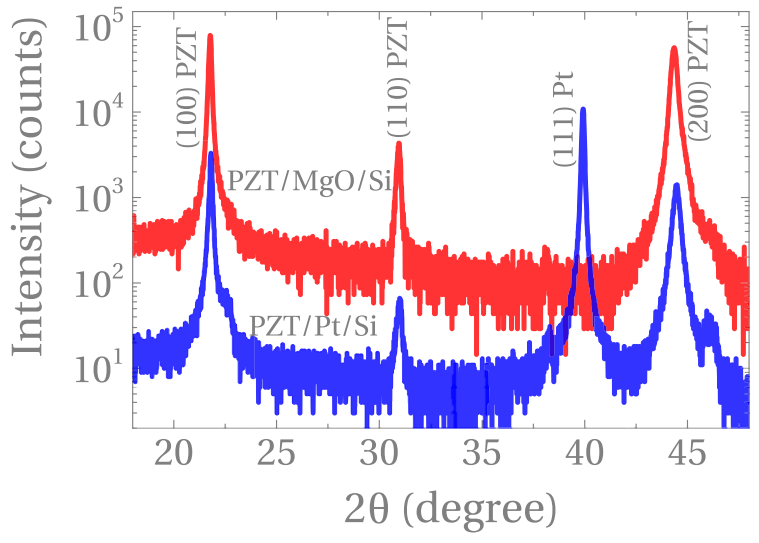

Figure 4. XRD pattern of $500 \mathrm{~nm}$ MPB gradient free PZT deposited on $\mathrm{MgO}$ buffered $\mathrm{Si}$ and on platinized Si substrate (with $20 \mathrm{~nm}$ of $\mathrm{PbTiO}_{3}$ seed layer).

Again the slope of stress with respect to electric field is $-e_{33, i f}$. For (100) Si substrate where longer axis of the cantilever is along [ 110$]$, the Young's modulus is $169 \mathrm{GPa}$ and Poisson's ratio is 0.064 [31].

\section{Results and discussion}

In this article, we propose a technique to obtain mixture of (100) and (110) oriented PZT with almost no (111) orientation, on insulator buffered silicon substrates which is ideally suited for IDE system. To get this desired orientation, we have employed $e$-beam evaporated $\mathrm{MgO}$ buffer layer, which prevent lead inter diffusion as well as promote (100) $\mathrm{PbTiO}_{3}$ nucleation. Figure 4 shows the XRD pattern of $500 \mathrm{~nm}$ gradient free MPB PZT deposited on $\mathrm{MgO}$ buffered Si substrate with a thin $\mathrm{PbTiO}_{3}$ seed layer along with $500 \mathrm{~nm}$ PZT deposited on platinized silicon. Figures 5(a) and $(b)$ shows the SEM top view and cross section of the PZT on $\mathrm{MgO}$ buffered silicon whereas figures 5(c) and $(d)$ show for PZT on platinized silicon. Both the films are dense and show columnar microstructure with no secondary phase on its surface.

Figure 6(a) shows the bright field TEM cross section of PZT deposited on $\mathrm{MgO}$. Here one can see the diffraction contrast in $\mathrm{MgO}$ barrier layer. Figure 6(b) shows the diffraction pattern from $\mathrm{MgO}$ and $\mathrm{PZT}$ region. The spots from $\mathrm{MgO}$ (highlighted in blue colour) are identified by comparing the diffraction pattern from PZT region alone. It is clearly seen that $\mathrm{MgO}$ (111) planes are oriented along the growth direction with some degree of tilt in the out of plane direction. This is independently confirmed with HRTEM data collected from the $\mathrm{PbTiO}_{3} / \mathrm{MgO}$ interface (not shown here). It is very interesting to note that like Pt (111), MgO (111) also promotes (100)/(001) $\mathrm{PbTiO}_{3}$ nucleation. In this work we have achieved over $95 \%$ (100) texture.

Simultaneous measurement of PV loop along with displacement data at $x_{2}$ (see figure 2) were performed on the cantilever test structures for IDE and PPE samples. Figure 7(a) shows the results of large signal polarization measurement performed at $20 \mathrm{~Hz}$ for IDE and PPE sample (IDE gap $a=11.8 \mu \mathrm{m}$, IDE finger width $b=3.3 \mu \mathrm{m}$, IDE width $W=1 \mathrm{~mm}$ and 100 

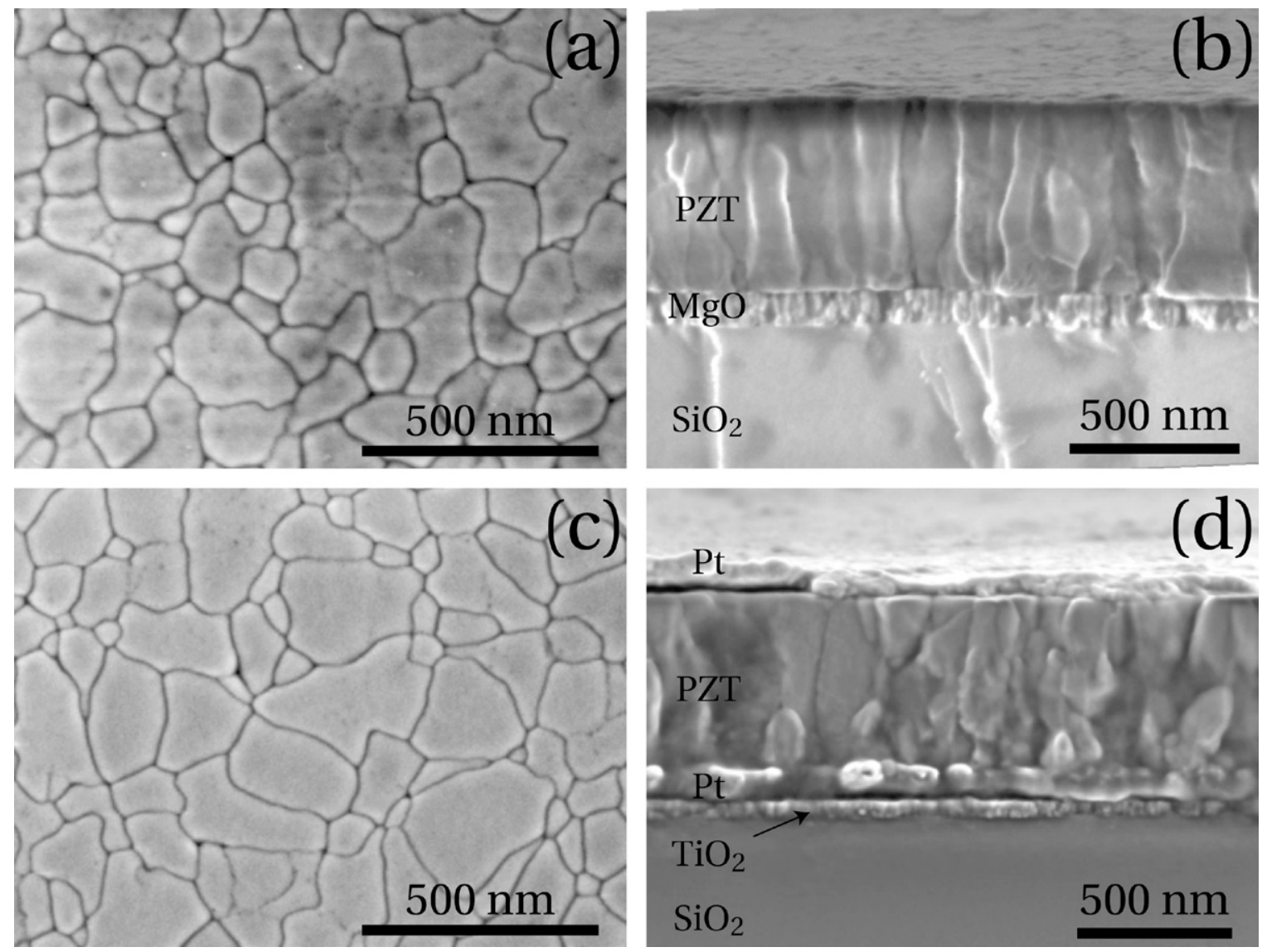

Figure 5. The SEM top view and cross section of PZT on $\mathrm{MgO}$ buffered Si substrate $((a)$ and $(b))$ and on platinized $\mathrm{Si}(c)$ and $(d)$.
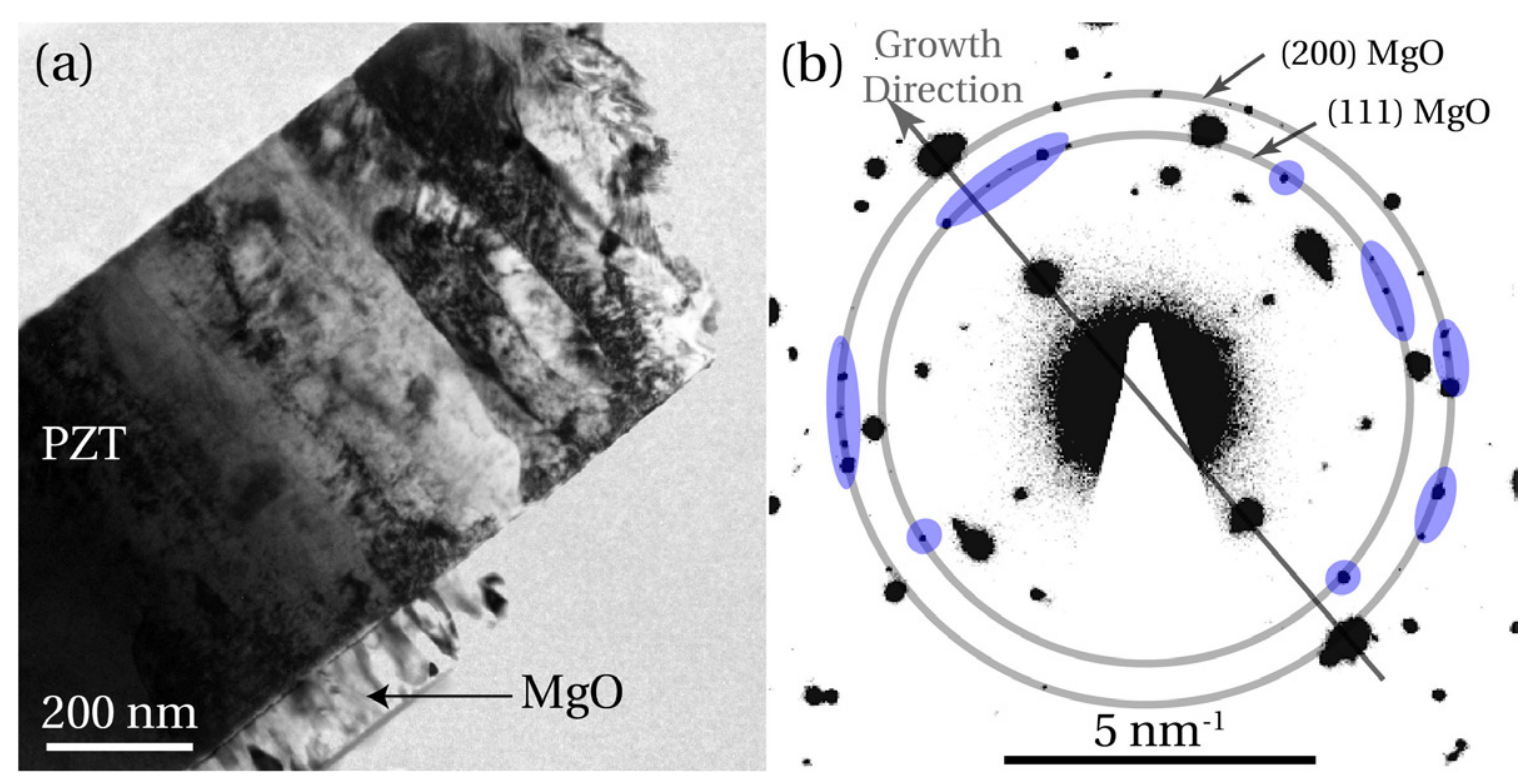

Figure 6. (a) TEM cross section of $500 \mathrm{~nm}$ PZT on $\mathrm{MgO}$ showing diffraction contrast in the MgO layer. (b) shows the diffraction pattern with the area selected for diffraction included both PZT and MgO. Translucent rings represent the (111) $\mathrm{MgO}$ plane and (200) MgO plane. Spots from $\mathrm{MgO}$ are highlighted in blue colour. It can be seen that the (111) $\mathrm{MgO}$ spots are seen along the growth direction with some degree of tilt.

pairs of IDE). Higher remnant and saturation polarization are observed for IDE sample. Figure $7(b)$ shows the $\left(1 / \epsilon_{0}\right)$ times the slope of PV curve, which indicates theoretical large signal permittivity. One can see high values (near switching fields) for IDE sample but similar values at high field conditions for both the IDE and the PPE case. From the tip displacement data, 


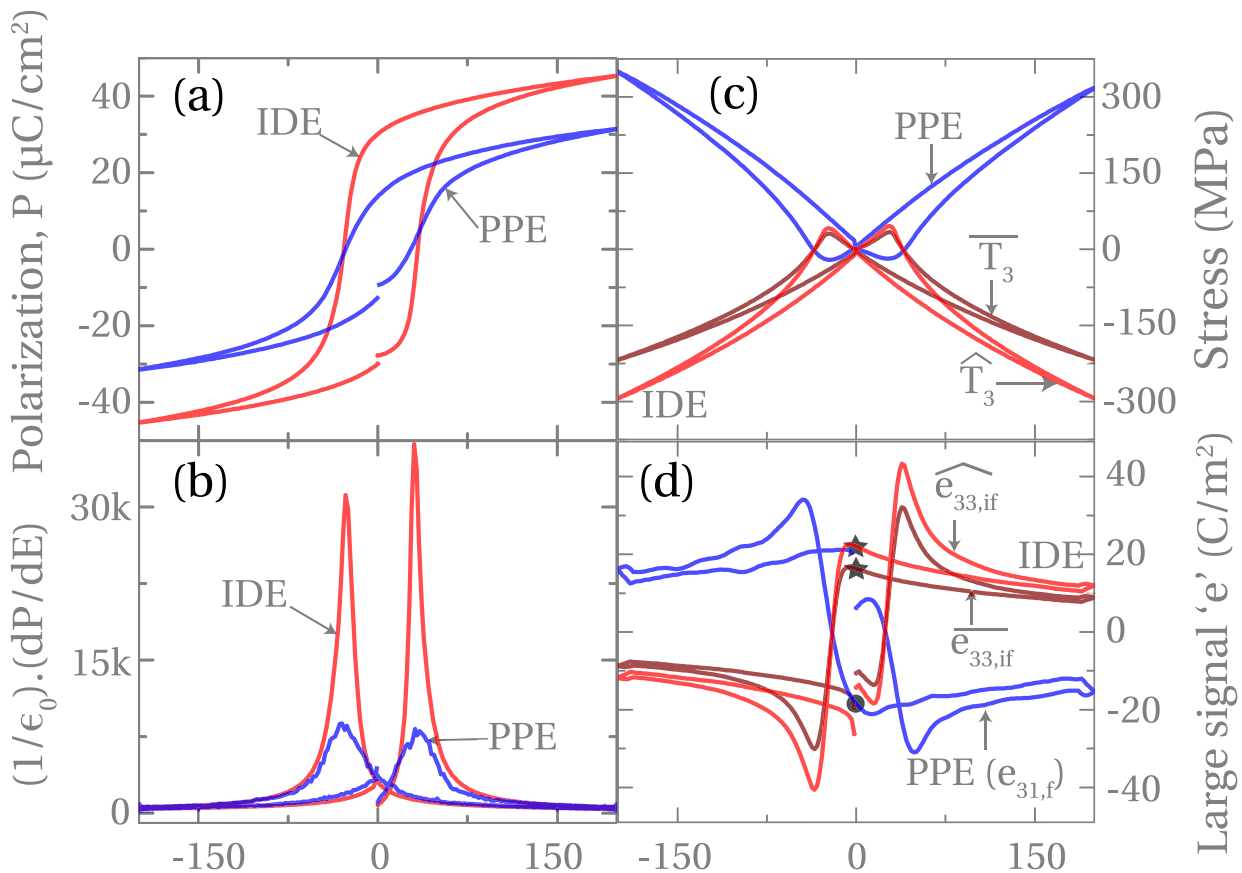

Electric field, $\mathrm{E}(\mathrm{kV} / \mathrm{cm})$

Figure 7. Large signal response for PPE (in blue) and IDE (in red and maroon) where red represents idealized stress and piezoelectric coefficient and maroon represents the average stress and piezoelectric coefficient. (a) polarization response (PV curve), $(b)$ slope of the PV curve or theoretical large signal permittivity, $(c)$ in-plane stress generated and $(d)$ derived large signal e piezoelectric coefficient.

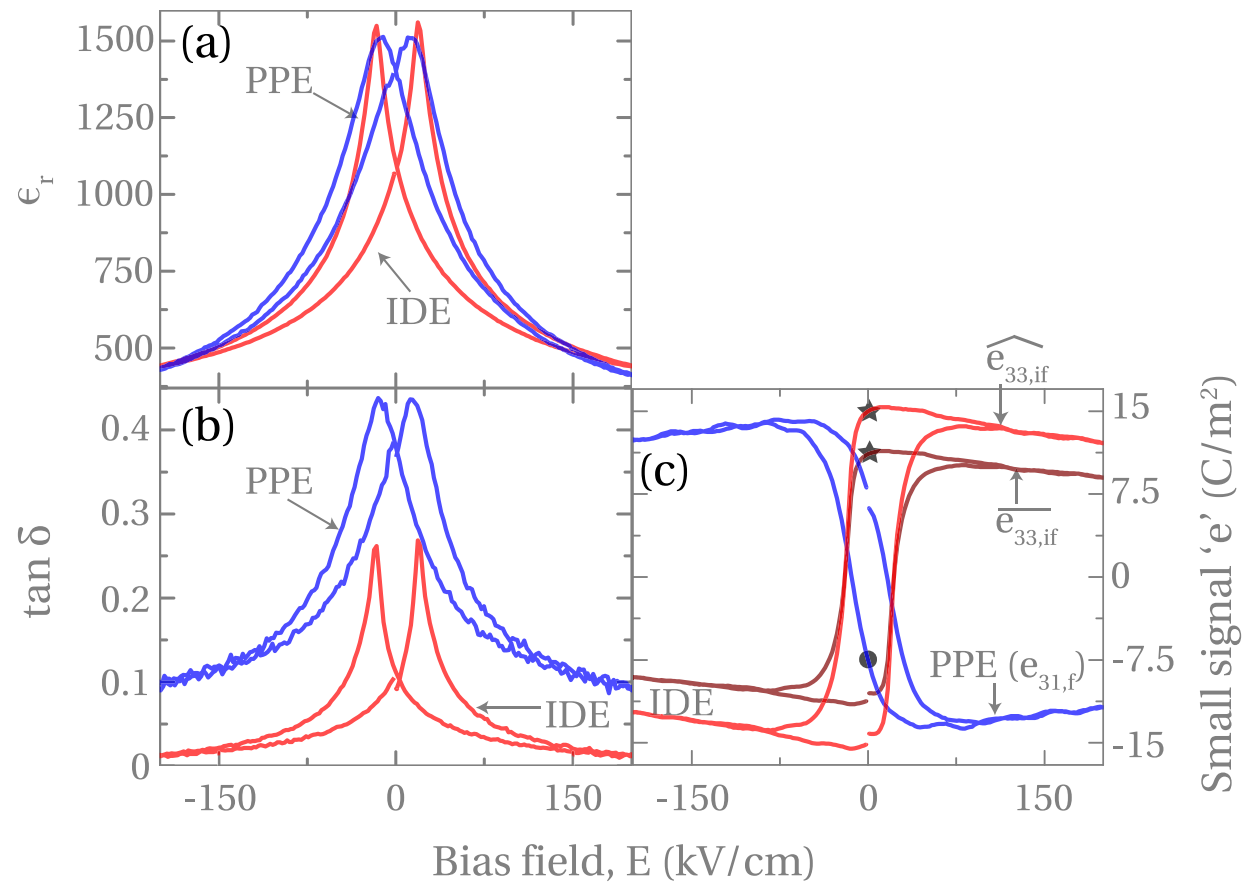

Figure 8. Small signal response for PPE (in blue) and IDE (in red and maroon) where red represents idealized stress and piezoelectric coefficient and maroon represents the average stress and piezoelectric coefficient. (a) Relative permittivity (CV curve), (b) losses and (c) small signal $e$ piezoelectric coefficient.

one can calculate the in-plane stress generated by the PZT film (figure $7(c)$ ) through the formula given in equations (16) and (18) for IDE case. For PPE case, $e_{31, f}$ coefficient was measured by taking the negative slope of in-plane stress developed with applied electric field. And the in-plane stress developed for PPE sample was derived to be the following [21]: 


$$
\begin{aligned}
T_{x} & =\frac{Y_{s}}{3\left(1-\nu_{s}\right)} \cdot \frac{h_{s}^{2}\left[c_{f}+\left(1-c_{f}\right)\left(1-\nu_{s}\right)\right]}{h_{f} c_{f}} \cdot \frac{u\left[x_{2}\right]}{x_{1}\left(2 x_{2}-x_{1}\right)} \\
& =-e_{31, f} \cdot E_{3}
\end{aligned}
$$

It is obviously seen that IDE geometry generates compressive piezoelectric stress whereas PPE geometry a tensile one. This is as expected, since the first one is dominated by the positive longitudinal coefficient $e_{33}$ and second one by the negative transverse coefficient $e_{31}$ (both define for a poled ceramics with field along the 3 direction). The negative slope of these stress curves are the large signal $e$ coefficients $\left(e_{33, i f}\right.$ for IDE and $e_{31, f}$ for PPE) shown in figure $7(d)$. For the film with IDE, average stress and piezoelectric coefficients and the idealized stress and piezoelectric coefficients are plotted. Simultaneous measurement of CV curve with small signal displacement were performed on the same cantilever test sample with a small signal of $1 \mathrm{kHz}, 10 \mathrm{kV} \mathrm{cm}^{-1}$ ac field with triangular bias swept at $0.1 \mathrm{~Hz}$. For the IDE case, the permittivity was calculated from the capacitance data along with the IDE dimensions using Schwarz-Christoffel transformation reported by Gevorgian et al [28]. Figures 8(a) and (b) shows the relative permittivity and losses for the IDE and PPE sample. Both the samples show similar permittivities, but lower losses for the IDE sample. Small signal $e$ coefficients are reported in figure $8(c)$ as a function of sweeping field. Both the films show similar coefficients at high fields, but close to zero field, higher values are measured for the IDE samples. This indicates a lesser back-switching, eventually combined with larger domain contributions in the IDE case. This observation is in fact compatible with higher polarization, steeper switching at $E_{\mathrm{c}}$ and a higher piezoelectric coefficient in the direct mode [13].

The coefficient in the converse mode, averaged between 0 to $200 \mathrm{kV} \mathrm{cm}{ }^{-1}$ amounts to $+15 \mathrm{C} \mathrm{m}^{-2}$ in the IDE case and $-15 \mathrm{C} \mathrm{m}^{-2}$ in the PPE case. The first value compares well with the $e_{33, i f}$, derived using the PZT 4 parameters (section 4: $e_{33, i f}$ of $16.3 \mathrm{C} \mathrm{m}^{-2}$ ). The same parameters set predicts a $e_{31, f}$ (PPE) of $-13 \mathrm{C} \mathrm{m}^{-2}$. We thus expected that $e_{33, \text { if }}$ should be $25 \%$ higher than $\left|e_{31, f}\right|$. However, they are about the same. The loss caused by the 'dead' zones of the electrode finger widths cannot be compensated. In our geometry, the IDE coefficient had an effective value (engineering coefficient) that was $25 \%$ lower than the PPE type. The value lower than expected for $e_{33, i f}$ might be due to grain boundary or grain diameter effects. As a matter of fact, the grains are much larger along the out-ofplane direction than in the in-plane direction (see figure 6(a)).

\section{Conclusions}

Converse mode piezoelectric $e$ coefficients for IDE systems have been derived from beam bending experiments $\left(e_{33, i f}\right)$. This idealized coefficient is independent of the nature of substrate unlike direct mode $e$ coefficient for IDE systems $\left(e_{\mathrm{IDE}}\right)$, which depends on the Poisson's ratio of the substrate. We compared directly IDE and PPE systems. For this purpose we grew highly (100) oriented, $500 \mathrm{~nm}$ thick PZT thin films on insulator buffered silicon substrates for the IDE samples. We introduced an insulating buffer layer of $\mathrm{MgO}$ (111), which is promoting (100) texture of our seed layer $\mathrm{PbTiO}_{3}$. The gradient free MPB PZT was deposited by a sol-gel technique. The reference film for PPE electrodes was grown by the same method, also in (100) texture and with the same thickness on a Pt (1 11 1) electrode. The dielectric, ferroelectric and piezoelectric responses were analyzed. Sweeping through the zero field, higher small signal piezoelectric coefficients were observed for the IDE system. In the actuator mode, however, at higher electric fields, the cantilever excursion was about 25 percent lower with the IDE. The piezoelectric coefficient between the electrode fingers was derived to be about the same size as the transverse one in the PPE configuration, which is less than expected. There is no gain with IDE actuator when going to high enough fields $\left(>E_{\mathrm{c}}\right)$. The advantage of IDE actuators was found to lie in their better polarization at small fields (less polarization back-switching), a lower hysteresis in the unipolar mode and a lower dielectric loss. Of course, the fact that the piezoelectric stress is compressive and not tensile, could give an advantage in reliability, as film cracking is much less likely. The work also confirmed our earlier observations that as sensor or harvester, the IDE geometry is more promising in terms of a better polarization stability, at least when using substrates with low thermal expansion like silicon.

\section{Acknowledgments}

The authors thank the Swiss National Science Foundation (FN 200020-138154 and FN 200020-150108/1) for funding this work.

\section{References}

[1] Okumura M and Takahashi T 2007 Novel micro piezo technology for ink jet printhead Prof. of the Int. Conf. on Digital Printing Technologies (Anchorage, Alaska, 18-21 September 2007) pp 314-8

[2] Kanno I, Kunisawa T, Suzuki T and Kotera H 2007 Development of deformable mirror composed of piezoelectric thin films for adaptive optics IEEE J. Sel. Top. Quantum Electron. 13 155-61

[3] Baran U, Brown D, Holmstrom S, Balma D, Davis W O, Muralt P and Urey H 2012 Resonant PZT MEMS scanner for high-resolution displays J. Microelectromech. Syst. 21 1303-10

[4] Wilke R H T, Johnson-Wilke R L, Cotroneo V, Davis W N, Reid P B, Schwartz D A and Trolier-McKinstry S 2013 Sputter deposition of PZT piezoelectric films on thin glass substrates for adjustable x-ray optics Appl. Opt. 52 3412-9

[5] Roundy S, Leland E S, Baker J, Carleton E, Reilly E, Lai E, Otis B, Rabaey J M, Wright P K and Sundararajan V 2005 Improving power output for vibration-based energy scavengers IEEE Pervasive Comput. 4 28-36

[6] Marzencki M, Basrour S, Belgacem B, Muralt P and Colin M 2007 Comparison of piezoelectric MEMS mechanical vibration energy scavengers 2007 NSTI Nanotechnology Conf. and Trade Show: NSTI Nanotech, Technical Proc. (Santa Clara, California, 20-24 May 2007) vol 3 pp 21-4

[7] Newns D M, Elmegreen B G, Liu X H and Martyna G J 2012 High response piezoelectric and piezoresistive materials 
for fast, low voltage switching: simulation and theory of transduction physics at the nanometer-scale $A d v$. Mater. 24 3672-7

[8] Muralt P, Kholkin A, Kohli M and Maeder T 1996 Piezoelectric actuation of PZT thin-film diaphragms at static and resonant conditions Sensors Actuators A 53 398-404

[9] Muralt P 1997 Piezoelectric thin films for MEMS Integr. Ferroelectr. 17 297-307

[10] White R M and Voltmer F W 1965 Direct piezoelectric coupling to surface elastic waves Appl. Phys. Lett. 7 314-6

[11] Bernstein J J, Bottari J, Houston K, Kirkos G, Miller R, Xu B Ye Y and Cross L E 1999 Advanced MEMS ferroelectric ultrasound 2D arrays Proc. of the IEEE Ultrasonics Symp. (Caesars Tahoe, NV, 17-20 October 1999) vol $2 \mathrm{pp}$ $1145-53$

[12] Chidambaram N, Mazzalai A and Muralt P 2012 Measurement of effective piezoelectric coefficients of PZT thin films for energy harvesting application with interdigitated electrodes IEEE Trans. Ultrason. Ferroelectr. Freq. Control 59 1624-31

[13] Chidambaram N, Mazzalai A, Balma D and Muralt P 2013 Comparison of lead zirconate titanate thin films for microelectromechanical energy harvester with interdigitated and parallel plate electrodes IEEE Trans. Ultrason. Ferroelectr. Freq. Control 60 1564-71

[14] Xu B, Ye Y, Cross L E, Bernstein J J and Miller R 1999 Dielectric hysteresis from transverse electric fields in lead zirconate titanate thin films Appl. Phys. Lett. 74 3549-51

[15] Song Z T, Chong N, Chan H L W and Choy C L 2001 Electrical and pyroelectric properties of in-plane polarized lead lanthanum titanate thin film Appl. Phys. Lett. 79 668-70

[16] Yu H G, Zou L, Deng K, Wolf R, Tadigadapa S and Trolier-McKinstry S 2003 Lead zirconate titanate MEMS accelerometer using interdigitated electrodes Sensors Actuators A 107 26-35

[17] Zhang Q Q, Gross S J, Tadigadapa S, Jackson T N, Djuth F T and Trolier-McKinstry S 2003 Lead zirconate titanate films for $d_{33}$ mode cantilever actuators Sensors Actuators A 105 $91-7$

[18] Hong E, Krishnaswamy S V, Freidhoff C B and Trolier-McKinstry S 2005 Micromachined piezoelectric diaphragms actuated by ring shaped interdigitated transducer electrodes Sensors Actuators A 119 521-7

[19] Hong E, Smith R, Krishnaswamy S V, Freidhoff C B and Trolier-McKinstry S 2006 Residual stress development in $\mathrm{Pb}(\mathrm{Zr}, \mathrm{Ti}) \mathrm{O}_{3} / \mathrm{ZrO}_{2} / \mathrm{SiO}_{2}$ stacks for piezoelectric microactuators Thin Solid Films 510 213-21

[20] Chun D-M, Sato M and Kanno I 2013 Precise measurement of the transverse piezoelectric coefficient for thin films on anisotropic substrate J. Appl. Phys. 113044111

[21] Mazzalai A, Balma D, Chidambaram N, Matloub R and Muralt P 2015 Characterization and fatigue of the converse piezoelectric effect in PZT films for MEMS applications J. Microelectromech. Syst. PP 1

[22] Dubois M A and Muralt P 1999 Measurement of the effective transverse piezoelectric coefficient $e_{31, f}$ of AlN and $\mathrm{Pb}\left(\mathrm{Zr}_{x}, \mathrm{Ti}_{1-x}\right) \mathrm{O}_{3}$ thin films Sensors Actuators A 77 106-12

[23] Prume K, Muralt P, Calame F, Schmitz-Kempen T and Tiedke S 2007 Piezoelectric thin films: evaluation of electrical and electromechanical characteristics for MEMS devices IEEE Trans. Ultrason. Ferroelectr. Freq. Control 54 8-14

[24] Wilke R H T, Moses P J, Jousse P, Yeager C and Trolier-McKinstry S 2012 Wafer mapping of the transverse piezoelectric coefficient, $e_{31, f}$, using the wafer flexure technique with sputter deposited Pt strain gauges Sensors Actuators A 173 152-7

[25] Gurkovich S R, Blum J B 1984 Preparation of monolithic lead-titanate by a sol-gel process Ultrastructure Processing of Ceramics, Glasses and Composites ed L L Hench and D R Ulrich (New York: Wiley) pp 152-60

[26] Ledermann N, Muralt P, Baborowski J, Gentil S, Mukati K, Cantoni M, Seifert A and Setter N 2003 \{100\}-Textured, piezoelectric $\mathrm{Pb}\left(\mathrm{Zr}_{x}, \mathrm{Ti}_{1-x}\right) \mathrm{O}_{3}$ thin films for MEMS: integration, deposition and properties Sensors Actuators A $105162-70$

[27] Calame F and Muralt P 2007 Growth and properties of gradient free sol-gel lead zirconate titanate thin films Appl. Phys. Lett. 90062907

[28] Gevorgian S S, Martinsson T, Linner P L J and Kollberg E L 1996 CAD models for multilayered substrate interdigital capacitors IEEE Trans. Microw. Theory Tech. 44 896-904

[29] Muralt P 2008 Recent progress in materials issues for piezoelectric MEMS J. Am. Ceram. Soc. 91 1385-96

[30] Stoney G G 1909 The tension of metallic films deposited by electrolysis Proc. R. Soc. Lond. A 82 172-5

[31] Hopcroft M A, Nix W D and Kenny T W 2010 What is the Young's modulus of silicon? Microelectromech. Syst. J. $19229-38$ 